\title{
Emotional Faces in Peripheral Crowding
}

\author{
Tian $\operatorname{Ran}^{1,2}$, Wenfeng Chen ${ }^{1}$, and Xiaolan $\mathrm{Fu}^{1, *}$ \\ ${ }^{1}$ State Key Laboratory of Brain and Cognitive Science, \\ Institute of Psychology, Chinese Academy of Sciences, \\ Beijing 100101, China \\ ${ }^{2}$ Graduate School of the Chinese Academy of Sciences, Beijing 100049, China \\ \{rant, chenwf, fuxl\} apsych.ac.cn
}

Crowding effect refers to the deficit in identifying viewed targets, such as letters, numerals, line segments, or grating patches, when other shapes are nearby. This effect is reduced when distractors have a different color, contrast, or binocular disparity than that of the target. With feature singleton targets, the crowding effect decreases dramatically with an increasing number of distractors for both simple orientation and more complex letter identification tasks [4]. With a target that is not a salient feature singleton, however, the increasing number of distractors worsens rather than improves the perception of the target [2].

The present study uses the popular paradigm of crowding effect to explore whether the emotional schematic faces are salient in the peripheral visual field, and can affect the crowding effect by bottom-up salience of target. For the emotional faces have the ability of attention capture [1][3], we expected to see a normal crowding effect with the target crowding by a single ring of distractors, and performance would be recovered as the distractors increasing. In other words, if the positive or negative expression can 'pop-out' in the parafovea, we expect to see, with emotional singleton targets, the crowding effect may decrease even with an increasing number of distractors (neutral schematic faces).

Three undergraduates ( 2 women and 1 man) were paid to participate in the experiment. Participants' task was to judge the emotional face (either positive or negative expression) among neutral expression is red or green and press the corresponding key.

A target was presented at a fixed eccentricity, $60 \mathrm{~mm}$ either to the left or to the right of the fixation point, which was at the centre of display (Fig. 1). From a viewing distance of approximately $57 \mathrm{~cm}$, this eccentricity corresponded to $6 \mathrm{deg}$ of visual angle. Distances (center to center) between rows and columns of objects were $16 \mathrm{~mm}$. Distractors (if present) formed a square matrix comprising $1 \times 1$ (a target alone), $3 \times 3,5 \times 5$, or $7 \times 7$ objects with the target in the center. Examples of stimuli for experiment were given in Fig. 2A, B, and C.

ANOVA of reaction times were conducted and the results showed that responses were fastest for no distractor condition $(M=255 \mathrm{~ms}$,

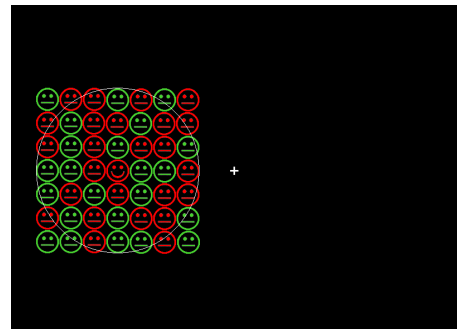

Fig. 1. An example of display

\footnotetext{
* Corresponding author.
} 
$S E M=6 \mathrm{~ms})$. A single ring of distractors caused the responses significantly slow down $(M=422 \mathrm{~ms}, S E M=6 \mathrm{~ms})$. And by increasing the number of distractors, response times were even slower, $496 \mathrm{~ms}$ for 24 distractors $(S E M=7 \mathrm{~ms})$ and $548 \mathrm{~ms}$ for 48 distractors $(S E M=7 \mathrm{~ms})$. The results of correct proportions showed the similar trend of results of reaction time, which suggests the perception of emotional faces might be affected by the distractors.

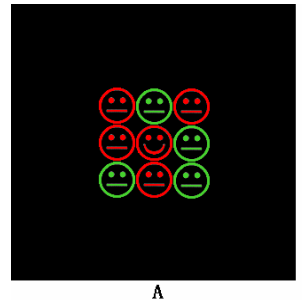

A

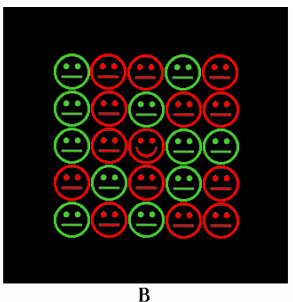

B

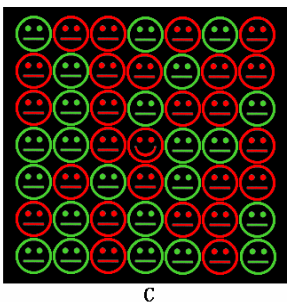

C

Fig. 2. Examples of stimuli used in the present study: (A) $3 \times 3$ matrix, (B) $5 \times 5$ matrix, and (C) $7 \times 7$ matrix. The stimuli were presented unpredictably either to the left or to the right of the fixation point (eccentricity $\approx 6 \mathrm{deg}$ ).

In the present experiment, the reaction time and accuracy data show that judgment is fastest and nearly perfect when there is a single ring of distractors, but as the increasing of rings of distractors, the judgment slows down with more errors. These are not consistent with the results of previous studies on crowding effect (e.g., [4]). The findings of this study suggest that the emotional features may not be very salient in the parafovea. This account requires further evidences to explore whether the recovered performance will be observed when the distractors increasing in the fovea.

\section{Acknowledgement}

This research was supported in part by grants from 973 Program (2006CB303101) and the National Natural Science Foundation of China (60433030, 30500157, and 30600182).

\section{References}

1. Dolan, R.J.: Emotion, cognition, and behavior. Science. 298, 1191-1194 (2002)

2. Felisberti, F.M., Solomon, J.A., Morgan, M.J.: The role of target salience in crowding. Perception 34, 823-833 (2005)

3. Hao, F., Zhang, H., Fu, X.: Modulation of Attention by Faces Expressing Emotion: Evidence from Visual Marking. In: Tao, J., Tan, T., Picard, R.W. (eds.) ACII 2005. LNCS, vol. 3784, pp. 195-201. Springer, Heidelberg (2005)

4. Poder, E.: Crowding, feature integration, and two kinds of "attention". Journal of Vision. 6, 163-169 (2006) 